\title{
NICE guideline: transient loss of consciousness (blackouts) in adults and young people
}

Greg Rogers and Norma O'Flynn

\section{INTRODUCTION}

Transient loss of consciousness (TLoC) is loss of consciousness with complete recovery. It is commonly described by the patient as a blackout. TLoC is very common and people who experience it may present to GPs during surgery hours or out of hours, or they may be referred from the ambulance services or the emergency department. There are a number of potential causes of TLoC:

- uncomplicated faint or situational syncope;

- orthostatic hypotension;

- dysfunction of the nervous system (epilepsy);

- dysfunction of the cardiovascular system (syncope); or

- dysfunction of the psyche (psychogenic attacks).

The diagnosis of the underlying cause is often inaccurate, inefficient, and delayed, and misdiagnosis is common. NICE guideline CG109 ${ }^{1}$ aims to define the appropriate pathways for the assessment of patients who experience TLoC, in order to obtain the correct underlying diagnosis quickly, efficiently, and cost-effectively, and to tailor a management plan to suit their true diagnosis. The guideline includes advice on the management of uncomplicated faint/situational syncope and orthostatic hypotension, as well as on detailed testing and assessment of those people considered to have a cardiovascular cause for their TLoC.

G Rogers, MSc, FRCGP, GP, The Bethesda Medical Centre, Margate. N O'FLynn, PhD, MRCGP, clinical director, National Clinical Guideline Centre for Acute and Chronic Conditions, Royal College of Physicians, London.

Address for correspondence

Dr Norma O’Flynn, National Clinical Guideline Centre for Acute and Chronic Conditions, Royal College of Physicians, 11 St Andrews Place, Regents Park, London NW1 4LE.

E-mail: norma.oflynn@rcplondon.ac.uk

Submitted: 3 September 2010;

final acceptance: 7 September 2010

OBritish Journal of General Practice 2011; 61: 40-42.

DOI: 10.3399/bjgp11X548965

\section{GUIDANCE}

\section{Initial assessment}

The guidance most relevant to GPs is likely to concern those areas related to initial assessment in order to determine whether the person has had TLoC, the possible causes of it, and the appropriate next steps.

History. Ask the person who has had the suspected TLoC, as well as any witnesses, to describe what happened before, during, and after the event. Try to contact, by telephone, any witnesses who are not present at the consultation. Record details about:

- circumstances of the event;

- person's posture immediately before TLoC;

- presence or absence of any prodromal symptoms (such as sweating or feeling warm/hot) and movement during event (for example, jerking of the limbs and duration);

- appearance (for example, whether eyes were open or shut) and colour of the person during the event;

- any biting of the tongue (record whether the side or the tip of the tongue was bitten);

- injury occurring during the event (record site and severity);

- duration of the event (onset to regaining consciousness);

- presence or absence during the recovery period of confusion or weakness down one side; and

- current medication that may have contributed to TLoC (for example, diuretics).

Ask also about details of any previous TLoC, including number of episodes and frequency, as well as the person's medical history and any family history of cardiac disease (for example, personal history of heart disease and family history of sudden cardiac death).

Examination. Perform examination as clinically indicated. For example:

- check and record vital signs (such as pulse rate, respiratory rate, and temperature) and lying and standing blood pressure, if clinically appropriate;

- examine for other cardiovascular and neurological 
signs, such as cardiac murmurs or neurological deficit, where relevant.

Electrocardiogram. It is recommended that everyone has a 12-lead electrocardiogram (ECG) recorded using automated interpretation. If any of the following abnormalities are present, referral within 24 hours for specialist cardiovascular assessment is recommended:

- conduction abnormality (for example, complete right- or left-bundle branch block or any degree of heart block);

- evidence of delayed atrioventricular conduction, including bundle branch block;

- evidence of a long or short QT interval; or

- any ST segment or T wave abnormalities.

If an automated ECG is not available, an ECG needs to be taken and interpreted by a trained and competent health professional who can identify the 'red flags' listed in Box 1.

The possibility of underlying problems that are either causing or contributing to TLOC should not be forgotten; relevant examinations and investigations may be required (for example, into blood glucose or haemoglobin levels).

\section{Differential diagnosis and management}

Cardiological causes. TLOC can occur due to an underlying cardiological problem. Referral for cardiovascular assessment within 24 hours is recommended if any of the following apply:

- an ECG abnormality;

- heart failure (history or physical signs);

- TLoC occurs during exertion;

- family history of sudden cardiac death in people aged $<40$ years and/or an inherited cardiac condition;

- new or unexplained breathlessness;

- a heart murmur.

TLoC occurring during exercise indicates that a cardiac arrhythmic cause is probable; it should be distinguished from TLoC that occurs shortly after stopping exercise, when a vasovagal cause is more likely.

Epilepsy. People who present with features that are strongly suggestive of epileptic seizures will require referral to a specialist in epilepsy. Features to note are:

- a bitten tongue;

- head turning to one side during TLoC;

- no memory of abnormal behaviour that was witnessed before, during, or after TLoC by someone else;
Box 1. Electrocardiogram 'red flags' that should prompt specialist cardiovascular assessment within 24 hours.

- Inappropriate persistent bradycardia

- Any ventricular arrhythmia (including ventricular ectopic beats)

- Long QT (corrected QT >450 ms) and short QT (corrected QT <350 ms) intervals

- Brugada syndrome ${ }^{a}$

- Ventricular pre-excitation (part of Wolff-Parkinson-White syndrome)

- Left or right ventricular hypertrophy

- Abnormal T wave inversion

- Pathological Q waves

- Atrial arrhythmia (sustained)

- Paced rhythm

${ }^{a}$ An inherited ion channel disorder, characterised by abnormal ST segment elevation in leads V1 to V3 on electrocardiogram. This predisposes the individual to ventricular arrhythmia and sudden cardiac death and may present with syncope.

- unusual posturing;

- prolonged jerking of limbs (note that brief seizure-like activity can often occur during uncomplicated faints);

- confusion following the event;

- prodromal déjà vu (whereby an unfamiliar situation feels familiar or is recognised) or jamais vu (whereby a familiar situation feels totally unfamiliar or is not recognised).

The episode may not be related to epilepsy if any of the following features are present:

- prodromal symptoms that, on other occasions, have been abolished by sitting or lying down;

- sweating before the episode or pallor during the episode; or

- prolonged standing that appeared to precipitate the TLOC.

Uncomplicated faint, situational syncope, and orthostatic hypotension. Uncomplicated faint (uncomplicated vasovagal syncope) should be diagnosed when there are no features that suggest an alternative diagnosis (note that brief seizure activity can occur during uncomplicated faints and is not necessarily diagnostic of epilepsy). Features suggestive of uncomplicated faint include:

- posture (prolonged standing or similar episodes that have been prevented by lying down);

- provoking factors (such as pain or a medical procedure); and

- prodromal symptoms (such as sweating or feeling warm/hot before TLoC).

These are known as 'the three Ps'. 
Situational syncope should be diagnosed when there are no features from the initial assessment that suggest an alternative diagnosis and syncope is clearly and consistently provoked by straining during micturition (usually while standing) or by coughing or swallowing.

If a diagnosis of uncomplicated faint or situational syncope is made, no further immediate management is required. The mechanism of the syncope, possible triggers, and avoidance strategies should be discussed and patients reassured. The guideline recommends that if the presentation is not to the GP, a copy of the patient report form and the ECG record should be taken to the GP or sent to the GP. If an ECG has not been recorded, the GP should arrange one within 3 days.

Orthostatic hypotension should be suspected on the basis of the initial assessment when there are no features suggesting an alternative diagnosis and the history is typical. If these criteria are met, measure the patient's lying and standing blood pressure (with repeated measurements while standing for 3 minutes). If clinical measurements do not confirm orthostatic hypotension despite a suggestive history, refer the person for further specialist cardiovascular assessment. If orthostatic hypotension is confirmed, likely causes or contributing factors, such as diuretics, should be considered.

\section{Further assessment and referral}

The outcome from the initial assessment will be that all people with TLoC who do not have a firm diagnosis of uncomplicated faint, situational syncope, orthostatic hypotension, or symptoms suggestive of epilepsy should have a specialist cardiovascular assessment by the most appropriate local service. The aim is to categorise the TLoC as either caused by suspected structural heart disease, suspected cardiac arrhythmia, suspected neurally mediated, or unexplained. Specific guidance is given as to appropriate further investigation, depending on the suspected cause. The following tests are likely during specialist assessment:

- An ambulatory ECG is required to diagnose a suspected cardiac arrhythmia. The type chosen will depend, in particular, on the frequency of TLoC and will require, for example, 24- or 48-hour monitoring or external or implantable event recorders.

- People with structural heart disease may have several mechanisms for syncope and so should have investigations for arrhythmia, as well as consideration of orthostatic hypotension and neurally medicating syncope, in addition to cardiac imaging.

- TLoC during exercise requires urgent (within 7 days) exercise testing, unless there is a possible contraindication (such as suspected aortic stenosis or hypertrophic cardiomyopathy). The patient should refrain from exercise until further assessment.
- For people with suspected carotid sinus syncope and for those with unexplained syncope who are aged $\geq 60$ years, carotid sinus massage is the firstline investigation.

- For people with suspected vasovagal syncope for whom recurrent episodes of TLoC adversely affect their quality of life or represent a high risk of injury, a tilt test is recommended. This will assess whether the syncope is accompanied by a severe cardioinhibitory response (usually asystole).

- An ambulatory ECG is recommended for all people with unexplained syncope (including after negative carotid sinus massage test in those for whom this is appropriate). A tilt test is not recommended before ambulatory ECG.

Appropriate advice for people who experience TLoC is also included in the guidance. This involves advising them that they must not drive while waiting for a specialist assessment and explaining the fact that they will need to report to the Driver and Vehicle Licensing Agency following diagnosis. Consideration of safety at work may also be required. In addition, patients should be told what to do if they have another event before assessment is completed.

\section{CONCLUSION}

From a general practice perspective, the recommendation that all people who have a TLOC episode should have an ECG is likely to be the biggest change from current practice. The recommendation that an automated ECG should be carried out is based on evidence assessing automated interpretation versus clinician-read ECGs, and evidence that clinicians who were not regularly interpreting ECG traces were likely to be less accurate than those who were experienced in this interpretation. Details of the evidence, together with discussions of the guideline development group, can be found in the full guideline.

\section{Funding body}

This work was undertaken by the National Clinical Guideline Centre for Acute and Chronic Conditions, which received funding from the National Institute for Health and Clinical Excellence (NICE). The views expressed in this publication are those of the authors and not necessarily those of NICE.

\section{Competing interests}

Greg Rogers is a GP and was a member of the guideline development group that formulated the guidance. Norma O'Flynn is clinical director at the National Clinical Guideline Centre (NCGC) where the guideline was developed. The NCGC is funded by NICE.

\section{Discuss this article}

Contribute and read comments about this article on the Discussion Forum: http://www.rcgp.org.uk/bjgp-discuss

\section{REFERENCE}

1. National Institute for Health and Clinical Excellence. Transient loss of consciousness ('blackouts') management in adults and young people. NICE clinical guideline 109. London: NICE, 2010.

http://guidance.nice.org.uk/CG109/NICEGuidance/pdf/English (accessed 29 Nov 2010). 